\title{
Nonlinear dynamics and self-organization of rotary molecular motor ensembles
}

\author{
Edeltraud Gehrig* and Ortwin Hess \\ Advanced Technology Institute, School of Electronics and Physical Sciences, University of Surrey, \\ Guildford, Surrey, GU2 7XH, United Kingdom \\ (Received 3 September 2005; revised manuscript received 27 March 2006; published 23 May 2006)
}

\begin{abstract}
The coupled nonlinear dynamics of rotary motor ensembles and an embedding fluid medium are simulated on the basis of Navier-Stokes equations. The model description considers space-dependent rotation of motors and a spatially inhomogeneous motor distribution as well as spatial fluctuations in molecular properties. The mutual influnce of motor rotations and fluid dynamics as well as spatial inhomogeneities of their molecular properties are self-consistently included. Space-time simulations visualize the complex interplay between the fluid dynamics and motor rotation and show that the dynamic coupling to the environment via the velocity field as well as the spatial inhomogeneity and distribution of rotors determines the overall behavior of the motor complex. Depending on the initial molecular distribution, rotation frequency, and fluid dynamics a spatial self-organization or a chaotic behavior may arise.
\end{abstract}

DOI: 10.1103/PhysRevE.73.051916

PACS number(s): 87.16.Nn, 87.10.+e, 05.40.-a

\section{INTRODUCTION}

Molecular motors are nanometer-sized functional biomolecular structures that consume energy to induce motion and to generate forces. Their dynamics is highly nonlinear and covers various time and length scales. Nature offers a large variety of different motor families characterized by a particular chemical structure, an environment, and a specific way of controlled (linear or rotary) motion. In the human body motor proteins control many essential processes such as cell division, transmembrane transport of ions and macromolecules, intracellular transport of vesicles and organelles, synthesis of ATP or DNA, muscle movement, or functioning of cells. Consumption of energy then leads to controlled translational (e.g., kinesin, myosin) or rotary motion of these molecular structures. Examples of rotary motors are the bacterial flagellar motor that rotates a flagellum using proton flow through the motor as the energy source and the F0F1-ATP synthase, which comprises two rotary motors, one driven by proton flow (F0 motor) and the other by ATP hydrolysis (F1 motor) [1,2]. Rotational motors play a key role in many biological membranes $[3,4]$. Additionally, due to their promising characteristics of working extremely efficiently and being controllable, artificial nanomotors can be conceived that directly employ the fundamental principals of biological motors [5]: The orientation of micrometer-sized particles could be driven and controlled by light [6,7]. Macroscopic magnetic disks (on a water surface) could be forced by a magnet to rotational motion [8]. Similarly, ATP-synthase could be used as a propeller inducing rotational motion in artifical machinery. This will open the way to novel micro- and nanotechnical devices such as particle separators or machines intermingling fluids that are based on and use the principles and mechanisms of these microscopic engines.

A main difference between biological and biotechnological rotary motor complexes lies in their size: Typical motor

\footnotetext{
*Electronic address: e.gehrig@surrey.ac.uk
}

densities in the chloroplasts of plants or in the mitochondriea of human cells are 1000 ATP-synthase motors per $\mu \mathrm{m}^{2}$ [9]. In contrast, artificial rotary motor complexes may be composed of larger particles, e.g., millimeter-sized magnetic disks on a water surface [10]. However, there are many characteristics that both types of systems have in common but it is, in particular, the dynamic coupling of spatially inhomogeneous motor ensembles and an environmental fluid that determines their overall behavior (e.g., assembly of cells or macroscopic particles). The complex behavior of natural biomolecular and novel biotechnological systems thus represents a formidable challenge for theoretical descriptions and numerical approaches that aim at a fundamental analysis of the underlying interaction mechanisms as well as interpretations or even control of a system's behavior.

In this paper we investigate the spatiotemporal dynamics of rotary motors interacting with an environmental fluid. Our theory is based on a mesoscopic, spatiotemporally resolved approach that bridges theoretical descriptions of microscopic biochemical properties of biological media with macroscopic system theories. The model takes into account, in particular, the various time and length scales and the full complexity of the interplay of spatial with temporal degrees of freedom. A space-dependent parameter set allows for a very general inclusion of molecular properties, particle distributions, and fluctuations. We use a classical approach for the rotating motor ensemble and couple their dynamics self-consistently with spatially and temporally resolved Navier-Stokes equations for the simulation of the fluid dynamics. The explicit consideration of the spatial dependence of the quantities represents an extension of spatially averaged studies based on the Navier-Stokes equations. Our spatially and temporally resolved integration of the Navier-Stokes equation thereby leads to a very natural and automatic inclusion of each motor-motor interaction.

In the following, we will describe and simulate the spatiotemporal dynamics of rotors embedded in a hydrodynamic fluid. The theory and modeling will include, in particular, the hydrodynamic motion in the fluid induced by the spatially distributed rotating motors as well as the influence of the 


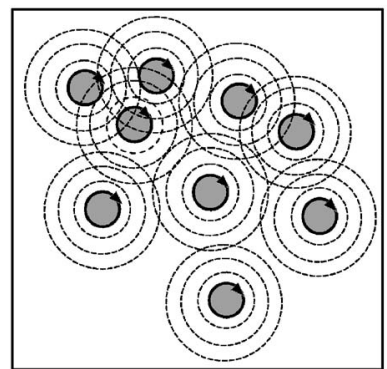

(a)

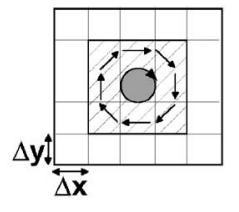

(b)
FIG. 1. (a) Scheme of the rotary model system. The spatially distributed rotating motors are embedded in an environmental medium. The dotted lines and arrows indicate the velocity field. The embedding fluid is initialized with a zero velocity field and then changes due to the coupling to the motor ensemble. (b) Simulation of the velocity distribution at the rim of each motor: the velocity fields in the neighboring meshes of the numerical grid (shaded area) are calculated according to the motor rotation.

flow dynamics on the rotation and spatial distribution of the motor ensemble.

\section{THEORETICAL DESCRIPTION}

We consider the situation displayed in Fig. 1(a). Rotary motors with individual positions, rotation frequencies, and locally varying coupling to the environment are spatially distributed within an embedding fluent medium. The rotation of the rotors generates a characteristic velocity field $v(r, t)$ in their environment. Thereby, the dynamics of each motor influences-via the mutual coupling of motors and the embedding fluent medium - the motion of the other motors. In the following we will derive a spatially and temporally resolved approach that includes the spatiotemporal dynamics of motors and their environment on a mesoscopic scale. The theory is based on the Navier-Stokes equations (for the simulation of the fluent medium) [9] that are dynamically coupled to a spatially inhomogeneous rotating motor ensemble. In principle, the motors may be driven by, e.g., a chemical reaction or an external force. Without loss of generality we will in the following refer to a motor ensemble that is driven by an external excitation. Thereby we focus our study on a classical rotation of monopolar particles (approximated by spheres), i.e., we neglect that the rotation (as in many biological motors) might occur in a stepwise motion. Furthermore, we do not consider dipolar motors (as, e.g., the ATP synthase). Our model system thus refers more to artificial motors that can be described by monopoles driven by an external field while biological macromolecules are driven by internally generated forces and correspond to rotational dipoles (as explained in, e.g., [11]). The (rotational and translational) dynamics of these motors is then determined by the angular frequency applied to each motor and the velocity distribution in the environmental fluid. The dynamics of the fluid, on the other hand, is strongly influenced by the rotation of the spatially distributed motors. We consider (without loss of generality) a system of classical, spherical particles of radius $R$ rotating with a uniform rotation. We note that an extension of the model description to a motor ensemble with a stepwise rotation or angle-dependent fluctuations (as, e.g., in the biological rotary motor F1-ADP synthase [4]) is straightforward. The motor distribution is given by $\rho_{m}(\mathbf{r}, t)$ where $\rho_{m}(\mathbf{r}, t)=\rho_{m}^{0}(\mathbf{r}, t)$ when a motor is located at position $\mathbf{r}$ in the biological medium and zero elsewhere. The motors are embedded in a Newtonian fluid characterized by the viscosity $\eta$ and density $\rho_{f l}$. The dynamics of the velocity field $v(\mathbf{r}, t)$ in the flow medium can be described by the following Navier-Stokes equations:

$$
\rho_{f l} \frac{\partial}{\partial t} v(\mathbf{r}, r)+\rho_{f l}[v(\mathbf{r}, t) \cdot \nabla] v(\mathbf{r}, t)-\eta \nabla^{2} v(\mathbf{r}, t)=0 .
$$

Thereby we have disregarded the pressure tensor (no temperature gradient). A very convenient approach for a description of the influence of a motor rotation on a fluent is to neglect in Eq. (1) the terms that depend on the density of the environment (i.e., restricting oneself to systems with small Reynolds numbers). This assumption then leads to the Stokes equation with solution $v=R^{3} / r^{3} \omega \times r$ [12]. Aiming at a description of the full spatiotemporal dynamics of spatially inhomogeneous motor-fluent medium complexes we here explicitly consider the full spatial and temporal dependence of the physical quantities and numerically integrate Eq. (1) without further approximations - in space and time. The consideration of the full spatial dependence of the variables thereby self-consistently describes the motor rotation by directly transferring the velocity field (induced at the rim of the rotor spheres by the motor rotation) to the medium. The velocity distribution in the immediate environment of grid points occupied by motors [shaded area in Fig. 1(b)] is then given by $v=\omega \times r$ (using the no-slip condition, i.e., assuming that the tangential velocity component is zero at the rotor surface). The velocity dynamics in the remaining spatial positions (at each grid point and for each time step) is calculated using Eq. (1). At every grid point and for each time step, the velocity field $v$ is calculated on the basis of finite difference methods (on a numerical grid with regular mesh size) using the hopscotch [13] method as a general scheme.

The coupling between the rotors and fluent medium and, particularly, the gradual transfer of the rotation to the environment happens due to the dynamic coupling between neighboring fluent layers. This is automatically included via the spatial dependence of Eq. (1). Due to our explicit consideration of the spatial and temporal degrees of freedom within the molecular ensemble we only need to take into account - at each spatial point $\mathbf{r}=(x, y)$ - the coupling of the velocity field to the immediate neighboring elements of the numerical grid, i.e., at $(x \pm \Delta x, y \pm \Delta y)$ (where $\Delta x$ and $\Delta y$ denote the length and width of a numerical element, respectively). This "local" interaction is directly realized via the spatial derivatives in Eq. (1). The influence of the rotation of a motor on the environment and-via the motor-fluentmedium coupling - to the dynamics of the neighboring motors is thus automatically included.

In principle, the spatiotemporal dynamics of the velocity field may change the position of the spatially distributed motors. Depending on the coupling between the motors and the 


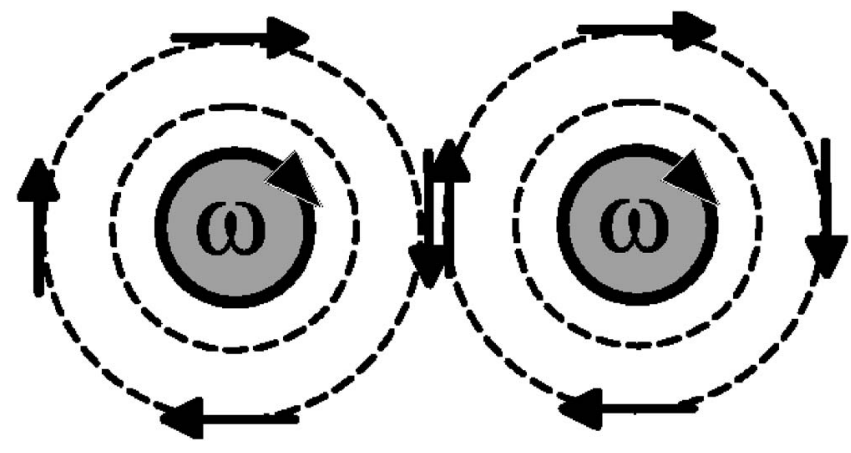

FIG. 2. Dynamic interaction between two motors: The dotted lines and arrows indicate the velocity fields.

environmental fluid (determined by, e.g., the viscosity and density) the motors may change their position. As a consequence, the distribution of the rotary motors has to be recalculated after each time step. In a final step, we thus include a possible change in motor positions induced by the dynamically varying velocity distribution. In general, the motors follow the local flow leading to dynamic positions of a new quasiequilibrium distribution. The new motor positions can thus be calculated by integrating Eq. (1) in space. In particular, this reorganization automatically includes the hydrodynamic repulsion between neighboring motors (as a direct consequence of inertial forces [10]) via their interaction in the embedding medium. The direct numerical integration of the partial differential equations of motion (1) allows a very general and straightforward representation of spatial inhomogeneities and nonequilibrium dynamics of the motor-fluentmedium system.

In the following, we will present selective results of our computational modeling based on this description. Without loss of generality, we will concentrate on motors characterized by a uniform (classical) rotation. We would like to note, however, that the integration of a stepwise rotation that often exists in biological motors (e.g., F1ATPase) is straightforward. Similarly, the theory can be extended to include biological motor complexes consisting of two rotors. In this case the velocity changes induced by the motors can be described by force centers at the edges of the motor complex [11] and the thickness of the membrane surrounding the motors has to be explicitly taken into account [12].

\section{SPATIOTEMPORAL DYNAMICS OF A TWIN-MOTOR SYSTEM}

In the following we will first concentrate on the dynamics of a twin-motor system (Fig. 2). The motors are immersed in a fluid of viscosity $\eta$, uniform density $\rho_{f l}$, and kinematic viscosity $\nu=\eta / \rho_{f l}$. The Reynolds number of the spinning spheres can be defined as $\operatorname{Re}=\omega R^{2} / \nu$. For the sizes used in our simulation $(R=400 \mu \mathrm{m})$ and rotation frequencies $\left.\left(\omega=0.5, \ldots, 5 \mathrm{~s}^{-1}\right)\right) \mathrm{Re}$ is of the order of $10^{-1}-1$, i.e., inertia plays an important role.

The two motors rotate with identical rotation vector $\omega=5 \mathrm{~s}^{-1}$ and radius $R=400 \mu \mathrm{m}$. They are positioned at a spatial separation of $D=5 \mathrm{~mm}$ witin an aqueous solution $\left(\eta=10^{-3} \mathrm{~kg} \mathrm{~m}^{-1} \mathrm{~s}^{-1} ; \rho=10^{3} \mathrm{~kg} / \mathrm{m}^{3}\right)$. Figure 3 shows snapshots of the motor distribution $\rho_{m}(r, t)(\mathrm{a})-(\mathrm{c})$ and the local kinetic energy $\varepsilon_{k i n}(r, t)=1 / 2 \rho_{f l} v^{2}(\mathbf{r}, t)(\mathrm{d})-(\mathrm{f})$ taken immediately after the start of the calculation (a), (d), and at $t=100$ (b), (e) and $200 \mathrm{~ms}$ (c), (f). At the start of the calculation, the motors were aligned parallel to the $y$ axis. The distribution of $\varepsilon_{k i n}(r, t)$ reveals the dynamics of the energy flow. Note that the abrupt cutoff in the center of the motor positions marks the finite spatial extension of the rotors. Immediately after the start of the calculation, the rotation of the two motors induces a dynamic velocity flow in the environment. Due to the finite rotation frequency and the viscosity of the environment this velocity field dynamically changes during the first rotation (corresponding to $200 \mathrm{~ms}$ for a rotation frequency of $5 \mathrm{~s}^{-1}$ ) until a quasiequilibrium state is reached. Similarly, the dynamic interplay of motor and fluent medium dynamics is still growing in this time window. We would like to note that the suitable design of such a velocity field around a set of motors may also be used to generate a specific flow to generate macroscopic motion. In [14] it was shown that F1ATPase motors can be integrated in functional nanoelectromechnanical systems to induce the rotation of nanopropellers. The simulation of such systems will be considered in future investigations. In the given example the motors are positioned rather close so that each motor experiences not only the velocity field induced by its own rotation but additionally the spatiotemporal changes in the velocity field induced by its neighboring motor. In the given example of two motors with identical rotational frequency, a spatial area of destructive interferences arises between the two motors. The interaction and mutual coupling thereby depends on

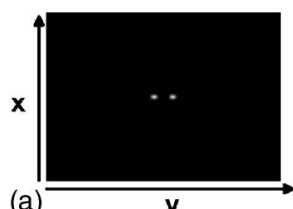

(a)

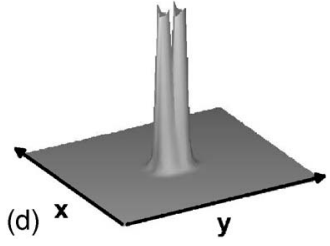

(b)
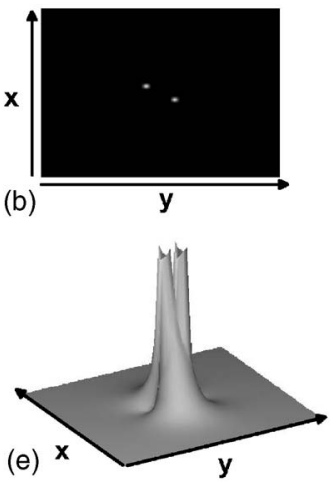

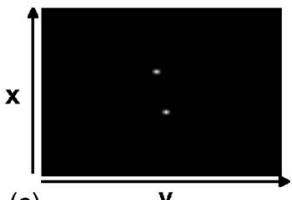

(c)

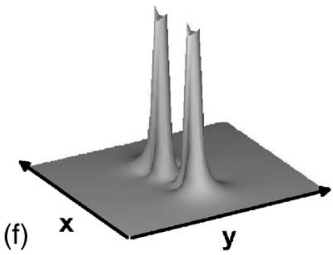

FIG. 3. Temporal snapshot of the spatial motor distribution (a)-(c) and the induced local energy flow (d)-(f) in a two-rotor system at $t=0$ (a), (d), 100 (b), (e) and $200 \mathrm{~ms}$ (c), (f). The initial spatial separation of the motors was $D=5 \mathrm{~mm}$, the rotation frequency $\omega=5 \mathrm{~s}^{-1}$. 


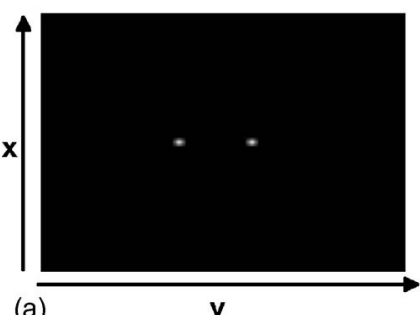

(a)

y

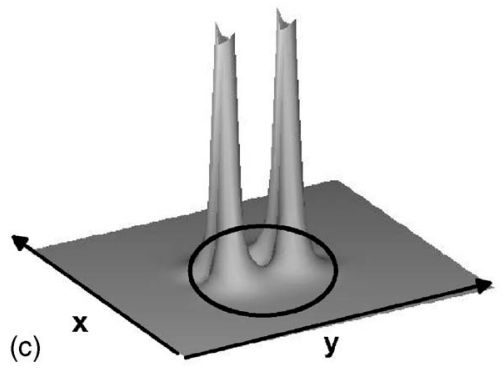

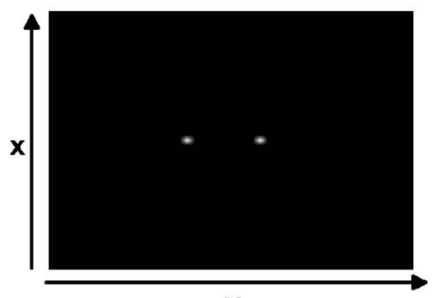

(b)

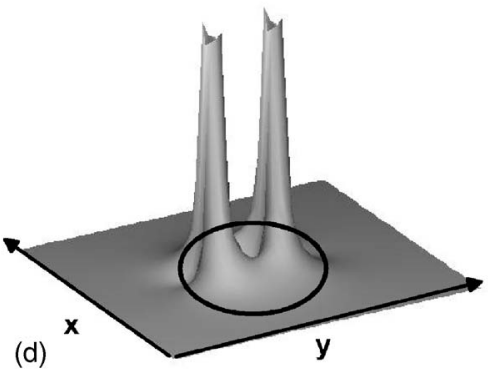

FIG. 4. Decoupled dynamics in a motor system with a spatial separation of $10 \mathrm{~mm}$ and a rotation frequency $\omega=5 \mathrm{~s}^{-1}$. The snapshots [taken at $t=100$ (a), (c) and $200 \mathrm{~ms}$ (b), (d)] show the distribution of the motors (a), (b) and the local energy distribution (c), (d) induced by the two motors. the rotation frequencies of the motors as well as on the physical properties of the environmental fluid. This effect can clearly be seen in the distribution of the local energy (being proportional to $v^{2}$ ) that dynamically evolves during the rotation of the motors. In the area between the two motors destructive interferences arises (Fig. 2) whereas constructive interference occurs in spatial regions where $|r|>D$. The corresponding kinetic energy consequently is smaller if $D$ is larger. The snapshots of the velocity and of the local energy distribution taken at $200 \mathrm{~ms}$ [Figs. 3(c) and 3(f)] shed light on two important effects. First, the motors experience a hydrodynamic repulsive force that pushes them apart. This translational shift directly originates from the dynamic spatiotemporal overlap of the velocity fields. In our model example it is, in particular, inertia that leads to the observed shift. Second, the motors may start a rotation around their center of mass following the energy flow of their environment. This effect can be understood by looking at the arrows indicating the overall velocity field around the motors sketched in Fig. 2 or by following the torque of the energy flow depicted in Fig. 3(f). The force pushing the motors apart is similar to the Magnus force which is well known from Bernoulli's equations in fluid dynamics where it is correlated with the pressure difference arising from a combined translational and rotational motion and consequently describes an inertial effect. The dissipative motor-fluent medium system given by our motor complex features an analog inertia effect via the viscosity. In real (viscoelastic) fluids, additional time scales may be present via the microscopic relaxation times of the components in the fluent medium [12]. The right-hand rotation of the two motors thus leads to a corresponding slow right-hand rotation of the motors around their center of mass [Fig. 3(c)]. The frequency of this collective rotation and the spatial separation of the two motors in the quasistationary case thereby depends on the properties of the fluent as well as on the initial separation and rotation frequency of the motors. If the motor separation exceeds a critical separation and/or the rotation frequency is too small to induce a significant change in the fluent then the motor interaction may be too small to induce a spatial drift. The snapshots taken at $t=100$ (a), (c) and $200 \mathrm{~ms}$ (b), (d) of the motor distribution and the local energy distribution induced by the two motors with a spatial separation of $1 \mathrm{~mm}$ and a rotation frequency of $\omega=5 \mathrm{~s}^{-1}$ shown in Fig. 4 are an example of such decoupled dynamics. With increasing time, a dynamic spatiotemporal coupling of the velocity fields arises leading to a dynamic overlap of the energy profiles [Fig. 4(d); the ellipse marks the small difference between the two time steps]. However, due to the larger separation, the dynamic spatial overlap is much smaller than in the case of the close motor formation (Fig. 3) and the influence of inertia on the motor dynamics is decreased. The two motors consequently continue their decoupled rotation without changing their positions. One should note that here we restricted our discussions to selective simulation results rather than exploring large parameter scans. We would like to note, however, that a large advantage of our model lies in the possibility of a systematic variation and visualization of the control parameters and the resulting distributions in a given motor system.

\section{DYNAMIC SELF-ORGANIZATION IN SPATIALLY INHOMOGENEOUS MOTOR ENSEMBLES}

Our computational modeling allows the description and simulation of spatially inhomogeneous motor systems. Due to the spatially and temporally resolved description we thus do not have to use, e.g., averaged quantities to describe the influence of the dynamics of the neighboring motors on a specific motor. The spatially and temporally resolved integration of the Navier-Stokes equation leads to a very natural and automatic inclusion of each motor-motor interaction. In the following discussion we will focus on a multimotor ensemble system embedded in an environmental fluid. Without loss of generality, we consider an ensemble consisting of $N=14$ motors. Depending on their spatial positioning and rotation frequency the motors may arrange themselves in the form of a regular grid, move about in a chaotic way, or appear completely decoupled. In the start of the calculation the motors (radius $400 \mu \mathrm{m}$ ) are initialized with a random spatial distribution in a spatial field of size $25 \times 25 \mathrm{~mm}^{2}$ 


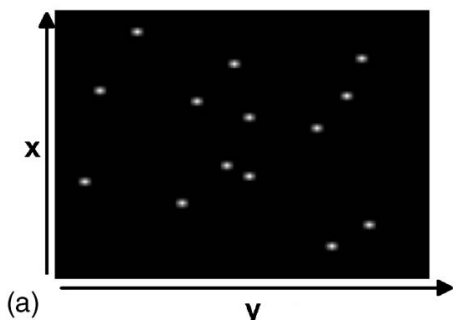

(a)

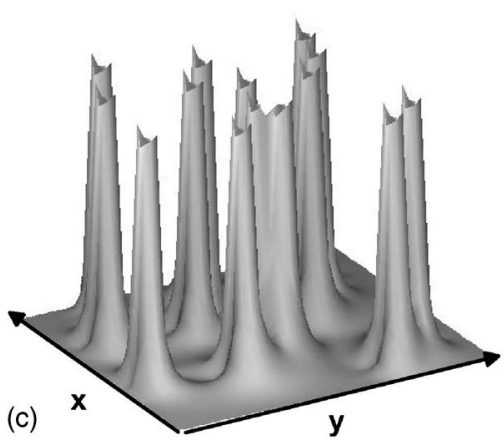

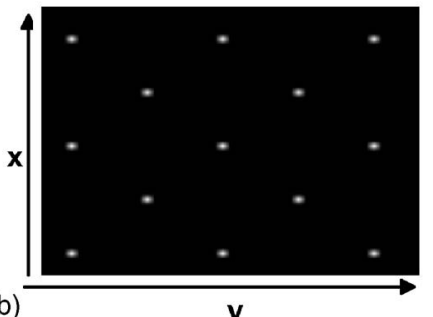

(b)

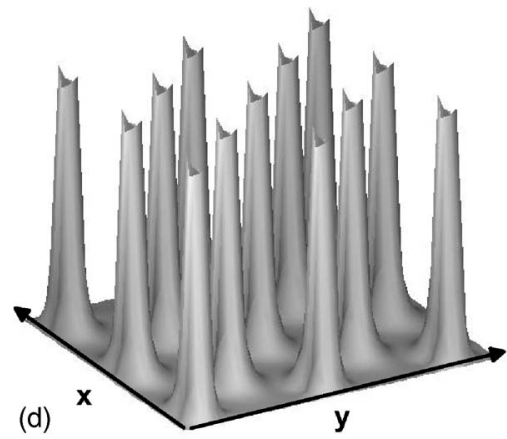

FIG. 5. Snapshots [at $t=200 \mathrm{~ms}$ (a), (c) and $2 \mathrm{~s}$ (b), (d)] of the motor distribution (a), (b) and local energy (c), (d) in a system consisting of $N=14$ motors $\left(\omega=5 \mathrm{~s}^{-1}\right)$.
[Fig. 5(a)]. We then study the following dependencies: the influence of a variation in the coupling strength between motors and environment (Sec. IV A) the influence of spatially inhomogeneity (Sec. IV B). Depending on rotation speed and fluctuations the motors may then reorganize in a crystalline order or form a disordered phase where diffusion is enhanced by velocity fluctuations.

\section{A. Influence of motor coupling}

In the following we will analyze the fundamentals of the interaction and coupling between neighboring motors. We will focus on the reorganization of strongly interacting motors as well as on the influence of rotation frequency and spatial separation of the motors. Figure 5 shows snapshots of the motor distribution (a), (b) and local energy (c), (d) in a system consisting of $N=14$ motors (all rotating in the same direction). Without loss of generality we consider a situation where the rotation frequency has been set to $\omega=5 \mathrm{~s}^{-1}$ and viscosity of the fluent to $\eta=10^{-3} \mathrm{~kg} \mathrm{~m}^{-1} \mathrm{~s}^{-1}$. The first snapshot (a), (c) is taken at time $t=200 \mathrm{~ms}$ after the start of the calculation where the rotating motors have already-due to their dynamical coupling to their environment-induced a significant overlap of their velocity fields and resulting energy distribution [Fig. 5(c)]. The collective behavior of the system in combination with the rotational motion of the motor molecules leads to a complex dynamics: The velocity field induced by each motor leads to a repulsive translational force acting on the neighboring motors. At the same time, each motor experiences the velocity field generated by the other motors. The dynamics of the motor ensemble consequently is determined by both the coupling of the motors to their environment and the physical properties of the fluent. The second snapshot (b), (d) is taken at a time point $t=2 \mathrm{~s}$ after the start of the calculation. In the shown example, the dynamic coupling between the rotating motors and their environment leads to a reorganization of the motor ensemble [Fig. 5(b)] in a gridlike structure (Wigner crystal) and to a corresponding minimization of the kinetic energy of the system [Fig. 5(d)]. The formation and stabilization of such a grating is strongly dependent on the coupling of the individual motors. As a consequence, a critical rotation frequency exists for a given motor arrangement [9]. For comparison, Figs. 6 and 7 show two examples that visualize the influencing factors of the rotation frequency (Fig. 6) and the
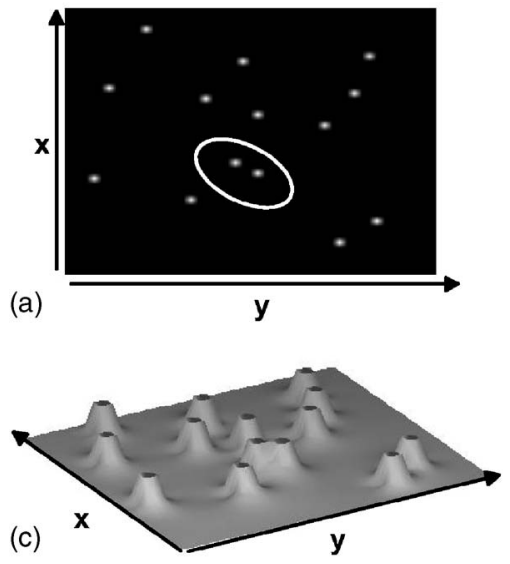

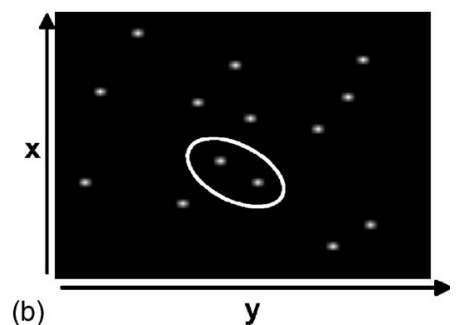

(b)

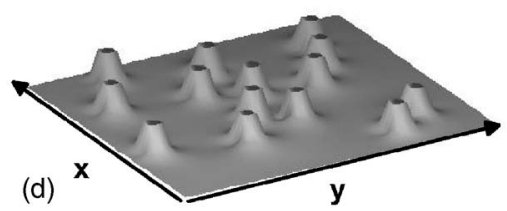

FIG. 6. Snapshots of the motor distribution (a), (b) and local energy (c), (d) for a rotation frequency of $\omega=1.0 \mathrm{~s}^{-1}$. The time steps and the scaling of the $z$ axis are the same as in Figs. 5(c) and 5(d). The white ellipse marks two motors that are slightly shifted in spite of the small motorfluent medium coupling. 


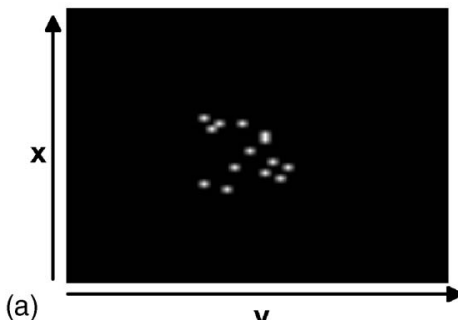

y

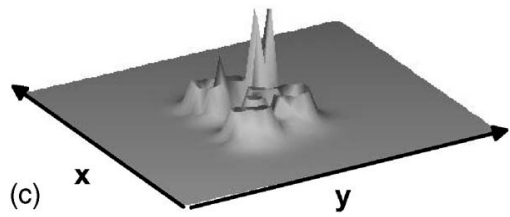

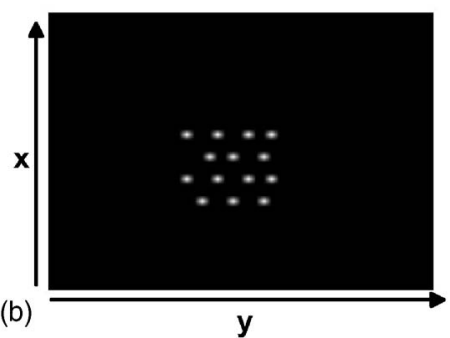

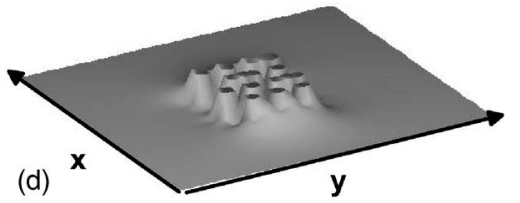

FIG. 7. Snapshots of the motor distribution (a), (b) and local energy (c), (d) $\left(\omega=1.0 \mathrm{~s}^{-1}\right)$ at $t=200 \mathrm{~ms}$ (a), (c) and $t=2 \mathrm{~s} \mathrm{(b),} \mathrm{(d)} \mathrm{in} \mathrm{a} \mathrm{system}$ where the motors are initially positioned in narrow spatial area near the center. initial spatial distribution (Fig. 7). Figure 6 shows-for the same initialization of the motor positions and the same time steps as in Fig. 5-snapshots of the motor distribution (a), (b) and local energy (c), (d) for a situation where the rotation frequency has been reduced to $\omega=1.0 \mathrm{~s}^{-1}$. In this case, the reduced velocity transferred from the motor rotation to the immediate motor environment leads to a smaller amplitude in the energy distribution [the scaling of the $z$ axis is the same as in Figs. 5(c) and 5(d)]. As a consequence, the changes in the energy distribution are to a larger degree restricted to the area immediately surrounding the motor positions. Only two motors with rather close initial positions [marked with a white ellipse in Figs. 6(a) and 6(b)] are slightly shifted apart while the remaining motors keep their initial positions.

For the same rotation frequency $\left(\omega=1 \mathrm{~s}^{-1}\right)$ Fig. 7 visualizes the influence of the initial spatial distribution. In this example, the motor positions were closer to the center of the rectangular spatial area. As a consequence, an increase in coupling strength arises due to the smaller average distance between neighboring motors and the motors again drift toward a regular gridlike structure in the second time step [Figs. 7(b) and 7(d)). In a given system, the lattice constant of the resulting grating is a function of initial distribution, rotation frequency, and the physical properties of the environmental fluent.

\section{B. Influence of spatial inhomogeneity}

The dynamics of the motor-fluent medium system depends not only on the coupling between the rotating motor ensemble and the environment but also on spatial inhomogeneities in the motor ensemble or fluent medium properties. Statistical fluctuations in the motor positions, rotation frequencies, or velocities may enhance a diffusive motion, thereby representing a counterpart of the reorganizing forces. In real motor systems the properties of the motors and of the fluent medium may strongly vary from one spatial point to another. In the following we will show results of simulations of a motor ensemble with variable rotation frequency, a spatially inhomogeneous fluent medium, and the generation of a macroscopic flow by inhomogeneous motor rotation. In the following we consider a motor ensemble with a spatially varying motor rotation. In our example we assume a spatial fluctuation of $10 \%$ (assuming a Gaussian distribution) around a central frequency of $\omega=1 \mathrm{~s}^{-1}$. Figure 8 shows two snapshots of the local energy distribution, taken at $t=200$ (a) and $2 \mathrm{~s}$ (b), respectively. The amplitude in the environment of the motor rims thereby directly reflects the spatially varying motor rotation that is transferred to the medium. One should note that in this case we have omitted the grid points directly neighboring the motor positions in order to plot the distribution of the velocity field only (without the velocity immediately at the rim given by the rotation frequencies). The motor-fluent medium coupling is rather moderate so that no reorganization of the spatial motor distribution occurs. However, a partial influence of the velocity distributions induced by the individual motors can clearly be seen in the second snapshot: The motors influence each other via their dynamic interaction with the fluent medium, leading to a partial influence of the velocity fields in the immediate environment of the individual motors. As a consequence, the

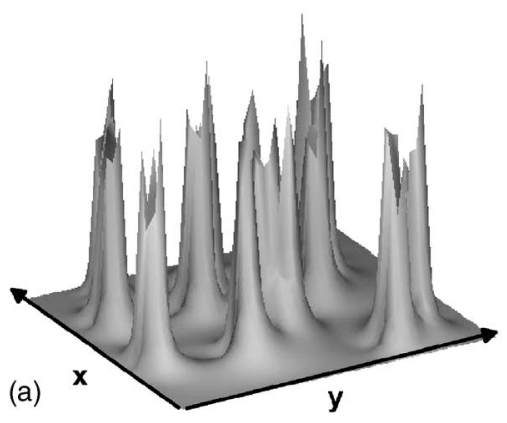

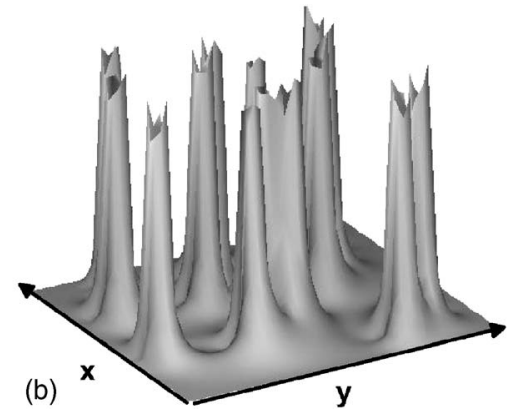

051916-6
FIG. 8. Snapshots of the local energy distribution at $t=200 \mathrm{~ms}$ (a) and $2 \mathrm{~s}$ (b) in a motor ensemble with a rotation frequency of $\omega=1 \mathrm{~s}^{-1}$ and a spatial flutation in $\omega$ of $10 \%$. 


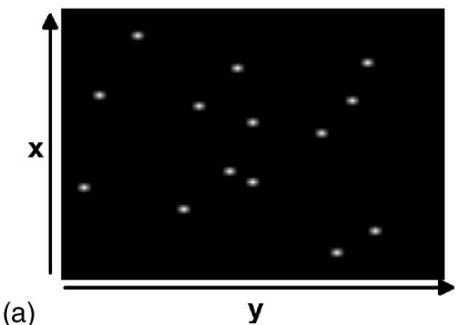

(a)

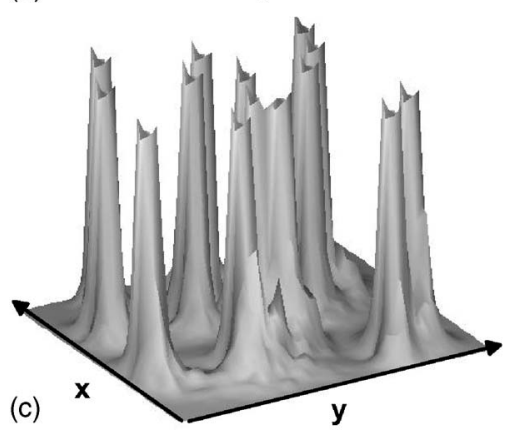

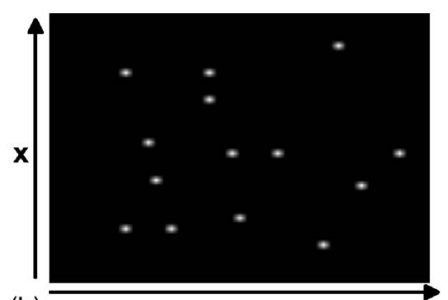

(b) y

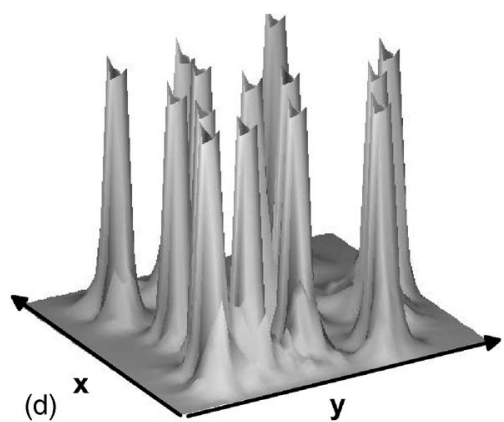

FIG. 9. Snapshots of the motor distribution (a), (b) and the local energy distribution (c), (d) for $t=1$ (a), (c) and $2 \mathrm{~s} \mathrm{(b),} \mathrm{(d)} \mathrm{in} \mathrm{a} \mathrm{motor-fluent}$ medium system with a spatial fluctuation in the fluent medium properties of $10 \%$. variation of the amplitude of the local energy near the rotor center becomes smaller with increasing interaction time [Fig. 8(b)].

In a next step we now consider a spatial inhomogeneity in the velocity field distribution. As an example, Fig. 9 shows the results of a simulation of a motor system with a spatial fluctuation in the velocity distribution of $10 \%$. Shown are snapshots of the motors [Figs. 9(a) and 9(b)] and the local energy fields [Figs. 9(c) and 9(d)] for $t=1$ (a), (c) and $2 \mathrm{~s} \mathrm{(b),}$ (d), respectively. Depending on the spatial arrangement and the physical properties of motors and the environment such flucutations may induce elongations of the motors from their quasiequilibrium and destabilize a given distribution. The snapshots of the motor distribution $\left(\omega=5 \mathrm{~s}^{-1}\right)$ [for $t=1$ (a) and $2 \mathrm{~s}(\mathrm{~b})]$ show that the motors dynamically change their positions but do not form a regular grid. The corresponding distributions of the local energy [Figs. 9(c) and 9(d)] visualize the fluctuations in the velocity field that lead to the dynamically changing motor positions. Depending on motorenvironment coupling and on the spatial separation from the neighboring motors the motor movements may increase or decrease with increasing time.

Recent investigations have demonstrated that a nonuniform rotation of motors (e.g., the vortical motion of cilia $[15,16]$ or that generated in a diffusive motor ensemble by using asymmetric boundaries [9]) may be responsible for left-right asymmetries in the vertebrate body plan. A nonuniform rotation may thus represent an interesting method for the generation of a macroscopic flow. Here we consider as an example a row of motors rotating with frequency $\omega=\omega_{0}+\delta \omega(\phi)$ where $\omega_{0}$ denotes the uniform contribution and $\delta \omega(\phi)=0.2 \sin (\phi)$ ( $\phi$ denotes the angle in the plane of the motor ensemble with respect to the motor row). Figure 10 shows a snapshot of the local energy for $\omega=0.5 \mathrm{~s}^{-1}$ and $t=200 \mathrm{~ms}$. The sketch on top of the distribution visualizes (for two rotors) the spatially inhomogeneous motor rotation. The nonuniform rotation introduces a spatiotemporal inhomogeneity in the system leading to dynamic (in the example sine-shaped) acceleration and deceleration of the motor rotation. As a consequence, the flow fields pointing to the left and the right sides of the motor row are of different magnitude leading to an overall flow in one direction (in our example, to the left side of the row; see sketch in Fig. 11). This example clearly demonstrates that an asymmetry or even control in a macroscopic flow may be generated by a suitable design of artificial motors. This result can only provide a first insight into possible control mechanisms or nanomechanical systems based on artificial motors. Future investigations will include details of the structure and movement of biological motors. This may then contribute to the interpretation of velocity fields in biological motor ensembles.

Our simulations on selective motor systems clearly reveal the complex dependence of a spatially inhomogeneous motor ensemble on rotation frequency, spatial distribution, and noise. The shown results cannot give more than a first indication of possible systematic simulations of real molecular systems. However, we would like to note that in particular the spatial and temporal resolution of the model description

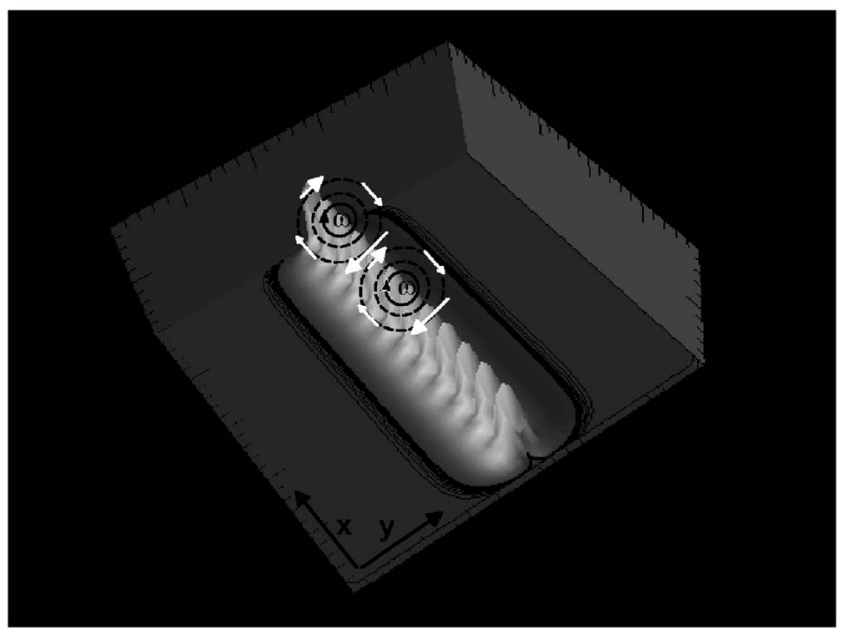

FIG. 10. Snapshots of the local energy distribution of a motor row with inhomogeneous rotation. 
as well as the explicit inclusion of spatial fluctuations in molecular properties (e.g., spatial ordering or rotation), noise, and dynamic motor-fluent medium coupling within the framework of the Navier-Stokes equations may, in principle, allow the systematic investigation of much more general motor-fluent medium systems. In particular, it may provide the basis for a fundamental and comparative analysis of both artificial motors driven by external fields and biological motors in a viscoeleastic solvent.

\section{CONCLUSION}

We have presented a spatially resolved Navier-Stokes model describing the coupled nonlinear dynamics of rotary molecular rotor ensembles within an embedding fluent. Our model includes, in particular, the coupling of the embedding fluid to a spatially inhomogeneous motor ensemble and extends exisisting models with respect to the spatial degree of freedom. Our mesoscopic approach is based on spatially resolved equations of motion describing the fluid dynamics and includes, in particular, space-dependent parameters for molecular properties and spatiotemporal noise. Space-time simulations on the basis of this approach reveal fundamental yet directly application-relevant results on the spatiotemporal dynamics of molecular motor complexes. They visualize a complex spatio-temporal interplay of motor ensembles and their environment and allow the fundamental analysis of the influence of particle distribution, angular frequencies of the rotary motion as well as spatiotemporal fluctuations in the physical parameters of the motors or the fluent. The explicit consideration of the spatial dependence allows the study and visualization of the influence of individual parameters and may be of particular importance for the realistic simulation of, e.g., a given experimental situation where spatial inhomogeneities in motor distribution and motor parameters are very typical. In our future investigations we plan to extend the model description to include the substructure and dynamics of complex biological motors such as, e.g., F1-ATPase. In particular, we plan to consider the dipolar character that is typical for many biological motors as well as the chemical reactions. This will allow the spatially resolved description of experimental observations such as, e.g., the artificial synthesis of ATP induced by mechanical energy [17] or the stepwise rotation in F1-ATPase [18]. The simulation of the full complexity of the spatially varying nonlinear fluent medium and motor dynamics may open new ways for fundamental analysis and potential control of collective behavior induced by the hydrodynamical interactions. Although we here have applied our studies to concrete systems the very general approach can, in principle, be applied to many other systems. In particular, the possibility to vary parameters individually may allow the prediction of potential control parameters that may enable an optimized efficient energy conversion and dynamics control. This may be of importance for both a physical understanding of fundamental biological processes in natural rotary motor complexes as well as the transfer into novel biotechnological devices.
[1] T. Nishizaka, K. Oiwa, H. Noji, S. Kimura, E. Muneyuki, M. Yoshida, and K. Kinosita, Jr., Nat. Struct. Mol. Biol. 11, 142 (2004).

[2] S. Pikin and W. Haase, J. Exp. Theor. Phys. 92, 174 (2001).

[3] J.-B. Manneville, P. Bassereau, S. Ramaswamy, and J. Prost, Phys. Rev. E 64, 021908 (2001).

[4] H. Noji, R. Yasuda, M. Yoshida, and K. K. Jr, Nature (London) 386, 299 (1997).

[5] R. Astumian, Sci. Am. 285, 56 (2001).

[6] P. Galajda and P. Ormos, Opt. Express 11, 446 (2003).

[7] P. Galajda and P. Ormos, Appl. Phys. Lett. 80, 4653 (2002).

[8] B. Grzybowski, H. Stone, and G. M. Whitesides, Nature (London) 405, 1033 (2000).

[9] P. Lenz, Phys. J. 3, 41 (2004).

[10] B. A. Grzybowski, X. Jiang, H. A. Stone, and G. M. Whitesides, Phys. Rev. E 64, 011603 (2001).
[11] P. Lenz, J.-F. Joanny, F. Jülicher, and J. Prost, Eur. Phys. J. E 13, 379 (2004).

[12] P. Lenz, J.-F. Joanny, F. Jülicher, and J. Prost, Phys. Rev. Lett. 91, 108104 (2003).

[13] I. S. Greig and J. D. Morris, J. Comput. Phys. 20, 64 (1976).

[14] R. K. Soong, G. D. Bachand, H. P. Neves, and A. G. Olkhovets, Science 290, 1555 (2000).

[15] S. Nonaka, H. Shiratori, Y. Saijoh, and H. Hamada, Nature (London) 418, 96 (2002).

[16] S. Nonaka, Y. Tanaka, Y. Okada, S. Takeda, A. Harada, Y. Kanai, M. Kido, and N. Hirokawa, Cell 95, 829 (1998).

[17] H. Itoh, A. Takahashi, K. Adachi, H. Noji, R. Yasuda, M. Yoshida, and K. Kinosita, Jr., Nature (London) 427, 465 (2004)

[18] R. Yasuda, H. Noji, M. Yoshida, K. Kinosita, Jr., and H. Itoh, Nature (London) 410, 898 (2001). 